\title{
Evaluation of bronchodilator responses in patients with "irreversible" emphysema
}

\author{
D.E. O'Donnell, L. Forkert, K.A. Webb
}

Evaluation of bronchodilator responses in patients with "irreversible" emphysema. D.E. O'Donnell, L. Forkert, K.A. Webb. C) ERS Journals Ltd 2001.

ABSTRACT: Given the emerging physiological and clinical rationale for pharmacological lung-volume reduction, assessment of volume responses to bronchodilators is likely to be highly relevant in chronic obstructive pulmonary disease (COPD). The authors examined the magnitude of lung-volume reduction after acute bronchodilator treatment in patients with advanced emphysema.

Eighty-four stable patients with emphysema (mean \pm SEM forced expiratory volume in one second (FEV1): $32 \pm 1 \%$ predicted) performed spirometry and body plethysmography before and 15-30 min after $200 \mu \mathrm{g}$ salbutamol. Only irreversible patients with a postbronchodilator change in $\mathrm{FEV} 1<\mathbf{1 0} \%$ pred were considered in this study.

Postsalbutamol, the majority of subjects $(83 \%)$ had significant improvements in one or more lung volumes: on average, residual volume (RV), functional residual capacity (FRC), inspiratory capacity (IC), forced vital capacity and slow vital capacity changed by $-18 \pm 2,-10 \pm 1,8 \pm 1,9 \pm 1$ and $7 \pm 1 \%$ pred $(p<0.0005$ each). Total lung capacity (TLC) decreased $0.12 \pm 0.04 \mathrm{~L}(\mathrm{p}<0.01)$. Change in IC reflected change in FRC $(r=-0.60, p<0.0005)$, but more strongly in the $57 \%$ of patients with no significant change in TLC $(\mathrm{r}=-\mathbf{0 . 9 3}, \mathrm{p}<0.0005)$. The magnitude and frequency of volume responses were greatest in patients with the most severe COPD; for example, RV decreased by $0.51 \pm 0.09 \mathrm{~L}(23 \pm 4 \%$ pred) and $0.27 \pm 0.04 \mathrm{~L}(14 \pm 2 \%$ pred $)$ in severe and moderate subgroups, respectively.

Significant reductions in lung hyperinflation occurred in the absence of a change in forced expiratory volume in one second after low-dose salbutamol in a majority of patients with advanced emphysema; the greatest changes occurred in those with the most severe disease.

Eur Respir J 2001; 18: 914-920.
Respiratory Investigation Unit, Dept of Medicine, Queen's University, Kingston, Ontario, Canada.

Correspondence: D.E. O'Donnell

Richardson House

102 Stuart Street

c/o Kingston General Hospital

Kingston

Ontario

Canada

K7L 2V7

Fax: 16135491459

Keywords: Bronchodilators

chronic obstructive pulmonary disease emphysema

inspiratory capacity

lung hyperinflation

reversibility criteria

Received: February 192001

Accepted after revision July 112001

This work was supported by the Ontario Ministry of Health.
The relatively diminished bronchodilator response in chronic obstructive pulmonary disease (COPD) (compared with asthma) has led to its designation as an irreversible airways disease and has, consequently, promoted a general attitude of therapeutic nihilism. Although the measurement of maximal flow rates e.g. the forced expiratory volume in one sec (FEV1) is of unquestionable diagnostic utility, and has become an acceptable (albeit imprecise) measure of disease severity in COPD, recent studies have shown that this measurement has definite limitations as a clinical outcome measure for the evaluation of bronchodilator efficacy [1-4]. In advanced COPD, forced expiratory manoeuvres initiated from total lung capacity (TLC) are fraught with measurement artefact (e.g. gas and airway compression effects) that underestimate the true maximal expiratory flows available over the operating tidal volume range [5]. Therefore, FEV1, only crudely reflects the degree of expiratory-flow limitation (EFL), which is the true pathophysiological hallmark of COPD [6]. The FEV1 correlates weakly, or not at all, with symptom intensity and exercise capacity in COPD [7-9].

A major consequence of EFL is air trapping and dynamic lung hyperinflation, which is arguably a relevant measure of impairment in severely flowlimited patients $[9,10]$. Symptom relief and improved exercise performance, following both $\beta_{2}$-agonist and anticholinergic bronchodilators, correlate well with reduced resting and exercise operational lung volumes in the presence of only minimal changes in the FEV1 $[1,2]$. Therefore, the purpose of this study was to examine the frequency and magnitude of lung-volume reduction in response to salbutamol in patients with irreversible emphysema. The authors were particularly interested in evaluating whether improved inspiratory capacity (IC) accurately reflected reduced lung hyperinflation in this population. The resting IC may be a clinically relevant outcome measure because it has recently been shown to correlate well with symptomlimited peak oxygen uptake in COPD $[10,11]$. Moreover, there is mounting evidence that small increases in IC (in the order of $\sim 10 \%$ predicted or $0.3 \mathrm{~L}$ ), following bronchodilator therapy are associated with important improvements in dyspnoea and exercise performance in COPD $[1,3,12,13]$. The behaviour of TLC following salbutamol was also studied, since this is essential to the evaluation of the potential utility of 
measuring changes in spirometric IC and vital capacity (VC) to accurately reflect changes in plethysmographic functional residual capacity (FRC) and residual volume (RV), respectively. Finally, the authors compared volume responses in patients with moderate (Stage II) and severe (Stage III) COPD, stratified by recent Global Initiative for Chronic Obstructive Lung Disease (GOLD) criteria [14].

In this study, the authors examined responses to $200 \mu \mathrm{g}$ of salbutamol in a large population of patients with well-characterized, stable, advanced COPD, who had clinical and physiological features of emphysema. The analysis was confined to those patients who had a minimal or absent FEV1 response during reversibility testing.

\section{Methods}

\section{Subjects}

The present authors studied 84 clinically-stable patients with advanced COPD, who did not meet the European Respiratory Society (ERS) reversibility criteria for a positive bronchodilator response [15]. Specific inclusion criteria included: 1) a clinical profile of emphysema [16]; 2) a long history of cigarette smoking ( $\geqslant 20$ pack-yrs); 3$)$ FEV $1 \leqslant 50 \%$ pred and FEV1/forced vital capacity (FVC) $<70 \%$; 4) lung hyperinflation (FRC $>120 \%$ pred); 5) a reduced carbon monoxide diffusing capacity of the lung $(D \mathrm{~L}, \mathrm{CO}) \leqslant 50 \%$ pred); and 6) a postbronchodilator FEV1 response $<10 \%$ pred. Subjects were further stratified with respect to COPD severity based on FEV1 criteria [14]: moderate (Stage II, FEV1 $\geqslant 30 \%$ pred and $\leqslant 50 \%$ pred) and severe (Stage III, FEV1 $<30 \%$ pred).

\section{Study design}

Subjects were selected from a database of COPD patients, who had performed reversibility testing during assessment before pulmonary rehabilitation or as part of the screening process prior to entering various clinical-research studies. All subjects signed written informed consent at the time of their original assessments and were aware that their test data might be used in future analyses. Prior to reversibility testing, patients were required to withdraw from all short-acting and long-acting bronchodilators for $\geqslant 4$ and $\geqslant 12 \mathrm{~h}$, respectively.

\section{Procedures}

Pulmonary function testing was conducted with subjects seated at rest, before and 15-30 min after $200 \mu \mathrm{g}$ of salbutamol metered dose inhaler with spacer. Spirometry [17] and constant-volume body plethysmography, with a panting frequency of $1-2 \mathrm{~Hz}$ [18], were performed using automated pulmonary function testing equipment (6200 Autobox DL or $V \max 229$ with 6200 Autobox; SensorMedics, Yorba
Linda, CA, US). Prebronchodilator only, singlebreath $D$ L, CO was also measured (6200 Autobox DL or $V \max 229)$. Predicted normal values for spirometry, lung volumes, and diffusing capacity were those of Morris et al. [19], CrApo et al. [20], and Burrows et al. [21], respectively. Predicted normal values for IC were calculated as predicted TLC minus predicted FRC.

\section{Evaluation of bronchodilator responses}

To establish eligibility for the study, bronchodilator responsiveness was first judged against FEV1 criteria recommended by the ERS [15]. To avoid bias from differences in baseline lung function [15], changes in various lung volumes were assessed and compared as $\%$ pred normal.

A change of $\geqslant 10 \%$ pred was felt to represent a significant bronchodilator response for IC or $\mathrm{VC}$, since this amount falls outside the $95 \%$ confidence interval (CI) for each of these measurements in this group and in other severe COPD populations [2, 22]. Based on the results from a previous study of patients with severe COPD, a change of this magnitude also fell outside the coefficient of variation for repeated measurements in this population [2]. In addition, it was estimated that an increase in IC of $10 \%$ pred, or $\sim 0.3 \mathrm{~L}$, resulted in clinically important improvements in exertional dyspnoea intensity (i.e. reductions of $\sim 0.5$ Borg Scale units) and in exercise endurance (i.e. increases of $\sim 25 \%$ ) in severe COPD.

Similarly, the proportion of patients with plethysmographic FRC and RV responses $>10 \%$ pred was evaluated. Finally, changes in TLC were also evaluated, and a significant change was assessed as a change outside the $95 \%$ CI of the within-group baseline measurement.

\section{Statistical analysis}

Results are means \pm SEM. Pre- and postbronchodilator comparisons were made using paired t-tests. Unpaired t-tests were used for subgroup comparisons. Nonparametric frequency statistics were analysed using Chi-squared analysis. Interrelationships between postbronchodilator changes $(\Delta)$ in lung function measurements were evaluated using Pearson's correlations. For subgroup comparisons and regression analyses, pulmonary function measurements were standardized as \% pred normal values.

\section{Results}

\section{Subject characteristics}

Subjects had significant airflow limitation, severe lung hyperinflation, a reduced $D \mathrm{~L}, \mathrm{CO}$, and a reduced body mass index (table 1). In a majority of patients, resting tidal flow/volume loops encroached on the maximal expiratory curve at isovolume. Concomitant respiratory medications included: short-acting $\beta_{2}$-agonists (100\% of patients), ipratropium bromide 
Table 1. - Subject characteristics

\begin{tabular}{|c|c|c|c|}
\hline & $\begin{array}{c}\text { All } \\
\text { subjects }\end{array}$ & $\begin{array}{l}\text { Moderate } \\
\text { COPD }\end{array}$ & $\begin{array}{l}\text { Severe } \\
\text { COPD }\end{array}$ \\
\hline Subjects $n$ & 84 & 48 & 36 \\
\hline $\mathrm{M}: \mathrm{F}$ & $60: 40$ & $50: 50$ & $72: 28^{*}$ \\
\hline Age yrs & $66 \pm 1$ & $68 \pm 1$ & $64 \pm 1^{*}$ \\
\hline Height $\mathrm{cm}$ & $165 \pm 1$ & $163 \pm 1$ & $168 \pm 1 *$ \\
\hline Weight kg & $64.9 \pm 1.6$ & $63.7 \pm 2.0$ & $66.5 \pm 2.4$ \\
\hline $\mathrm{BMI} \mathrm{kg} \cdot \mathrm{m}^{-2}$ & $23.7 \pm 0.5$ & $23.8 \pm 0.7$ & $23.5 \pm 0.7$ \\
\hline \multicolumn{4}{|l|}{ Pulmonary function } \\
\hline FEV1 L & $0.77 \pm 0.03$ & $0.86 \pm 0.04$ & $0.64 \pm 0.02$ \\
\hline \% pred & $32 \pm 1$ & $37 \pm 1$ & $24 \pm 1 * * *$ \\
\hline FEV1/FVC \% & $41 \pm 1$ & $43 \pm 1$ & $38 \pm 1$ \\
\hline$\%$ pred & $59 \pm 1$ & $62 \pm 2$ & $54 \pm 1 * * *$ \\
\hline FVC L & $1.91 \pm 0.07$ & $2.04 \pm 0.09$ & $1.75 \pm 0.08$ \\
\hline$\%$ pred & $55 \pm 1$ & $62 \pm 2$ & $46 \pm 2 * * *$ \\
\hline SVC L & $2.22 \pm 0.07$ & $2.30 \pm 0.09$ & $2.10 \pm 0.09$ \\
\hline$\%$ pred & $65 \pm 2$ & $71 \pm 2$ & $56 \pm 2 * * *$ \\
\hline IC L & $1.41 \pm 0.04$ & $1.51 \pm 0.07$ & $1.29 \pm 0.05$ \\
\hline$\%$ pred & $62 \pm 2$ & $69 \pm 2$ & $53 \pm 2 * * *$ \\
\hline TLC L & $7.42 \pm 0.17$ & $6.98 \pm 0.20$ & $8.01 \pm 0.27$ \\
\hline$\%$ pred & $137 \pm 2$ & $136 \pm 3$ & $139 \pm 3$ \\
\hline RV L & $5.21 \pm 0.15$ & $4.69 \pm 0.15$ & $5.91 \pm 0.24$ \\
\hline$\%$ pred & $253 \pm 6$ & $235 \pm 8$ & $276 \pm 9 * * *$ \\
\hline FRC L & $6.00 \pm 0.16$ & $5.47 \pm 0.18$ & $6.71 \pm 0.25$ \\
\hline$\%$ pred & $193 \pm 4$ & $185 \pm 6$ & $204 \pm 5^{*}$ \\
\hline $\begin{array}{l}\text { DL,CO, } \\
\mathrm{mL} \cdot \mathrm{min}^{-1} \cdot \mathrm{mmHg}^{-1}\end{array}$ & $7.5 \pm 0.2$ & $7.4 \pm 0.3$ & $7.5 \pm 0.4$ \\
\hline$\%$ pred & $37 \pm 1$ & $38 \pm 1$ & $36 \pm 2$ \\
\hline
\end{tabular}

Data are presented as mean \pm SEM unless otherwise stated. COPD: chronic obstructive pulmonary disease; M: male; F: female; BMI: body mass index; FEV1: forced expiratory volume in one second; FVC: forced vital capacity; SVC: slow vital capacity; IC: inspiratory capacity; TLC: total lung capacity; RV: residual volume; FRC: functional residual capacity; $D$ L,CO: carbon monoxide diffusing capacity of the lung; \% pred: percentage of predicted. FRC was plethysmographically determined. Subgroup comparisons were made between \% pred normal values only. *: $p<0.05$, $* * *: p<0.001$, significant difference between subgroups.

(74\%), inhaled corticosteroids $(70 \%)$, and oral theophylline $(13 \%)$. At the time of study, there were no patients using long-acting bronchodilators or oral corticosteroids.

The severe subgroup $(n=36)$ had significantly greater airflow obstruction and lung hyperinflation than the moderate subgroup $(n=48)$, with no difference between subgroups in DL,CO (table 1). On average, baseline IC was reduced at $62 \pm 2 \%$ pred, with a $95 \% \mathrm{CI}$ of $\pm 0.09 \mathrm{~L}$ or $\pm 3.7 \%$ pred. The moderate subgroup had a relatively well-preserved IC, with $50 \%$ of the subgroup having an IC $>70 \%$ pred; whereas, the severe subgroup had a significantly reduced IC, with only two patients with an IC $>70 \%$ pred (table 1).

\section{Bronchodilator responses}

Changes in response to salbutamol are provided in table 2 and figure 1. Although there was a statistically significant increase in FEV1 $(p<0.0005)$, there was no improvement, and actually a decline, in the
Table 2. - Responses to $200 \mu \mathrm{g}$ salbutamol metered dose inhaler

\begin{tabular}{lcrc}
\hline & Absolute $\Delta$ & $\Delta \%$ pred & $\Delta \%$ \\
\hline FEV1 L & $0.09 \pm 0.01^{\#}$ & $3.4 \pm 0.4$ & $12 \pm 1$ \\
FEV1/FVC \% & $-2.1 \pm 0.4^{\#}$ & $-2.9 \pm 0.7$ & $-5 \pm 1$ \\
FVC L & $0.32 \pm 0.03^{\#}$ & $9.2 \pm 0.7$ & $18 \pm 1$ \\
SVC L & $0.31 \pm 0.03^{\#}$ & $7.2 \pm 0.7$ & $12 \pm 1$ \\
IC L & $0.19 \pm 0.04^{\#}$ & $8.1 \pm 1.5$ & $15 \pm 3$ \\
TLC L & $-0.12 \pm 0.04^{* *}$ & $-2.5 \pm 0.7$ & $-2 \pm 1$ \\
RV L & $-0.37 \pm 0.05^{\#}$ & $-17.9 \pm 2.0$ & $-7 \pm 1$ \\
FRC L & $-0.31 \pm 0.05^{\#}$ & $-9.9 \pm 1.4$ & $-5 \pm 1$ \\
Severe subgroup & & & \\
FEV1 L & $0.10 \pm 0.01^{\#}$ & $3.7 \pm 0.5$ & $15 \pm 2^{*}$ \\
FEV1/FVC \% & $-2.4 \pm 0.7^{* *}$ & $-3.1 \pm 1.0$ & $-6 \pm 2$ \\
FVC L & $0.38 \pm 0.04^{\#}$ & $10.0 \pm 0.9$ & $23 \pm 2^{* *}$ \\
SVC L & $0.32 \pm 0.04^{\#}$ & $8.3 \pm 1.0$ & $16 \pm 2^{*}$ \\
IC L & $0.27 \pm 0.04^{\#}$ & $11.1 \pm 1.7^{\S}$ & $22 \pm 4^{* *}$ \\
TLC L & $-0.19 \pm 0.06^{* *}$ & $-3.2 \pm 1.0$ & $-2 \pm 1$ \\
RV L & $-0.51 \pm 0.09^{\#}$ & $-22.7 \pm 3.5^{*}$ & $-8 \pm 1$ \\
FRC L & $-0.45 \pm 0.08^{\#}$ & $-13.5 \pm 2.3^{*}$ & $-7 \pm 1^{*}$ \\
Moderate subgroup & & & \\
FEV1 L & $0.08 \pm 0.01^{\#}$ & $3.4 \pm 0.6$ & $10 \pm 2$ \\
FEV 1/FVC \% & $-1.8 \pm 0.5^{* *}$ & $-1.8 \pm 0.5$ & $-4 \pm 1$ \\
FVC L & $0.28 \pm 0.04^{\#}$ & $8.6 \pm 1.0$ & $15 \pm 2$ \\
SVC L & $0.21 \pm 0.04^{\#}$ & $6.4 \pm 1.0$ & $10 \pm 2$ \\
IC L & $0.14 \pm 0.05^{* *}$ & $5.8 \pm 2.2$ & $10 \pm 3$ \\
TLC L & $-0.08 \pm 0.05$ & $-1.9 \pm 1.0$ & $-1 \pm 1$ \\
RV L & $-0.27 \pm 0.04^{\#}$ & $-14.2 \pm 2.3$ & $-6 \pm 1$ \\
FRC L & $-0.21 \pm 0.06^{\#}$ & $-7.1 \pm 1.8$ & $-4 \pm 1$ \\
\hline Data a 0 & & & \\
\hline
\end{tabular}

Data are presented as mean \pm SEM. FEV 1 : forced expiratory volume in one second; FVC: forced vital capacity; SVC: slow vital capacity; IC: inspiratory capacity; TLC: total lung capacity; RV: residual volume; FRC: functional residual capacity; \% pred: percentage of predicted. ${ }^{\top}: \mathrm{n}=36$; ${ }^{+}: \mathrm{n}=48$. FRC was plethysmographically determined. *: $\mathrm{p}<0.05, * *: \mathrm{p}<0.01,{ }^{\#}: \mathrm{p}<0.0005$, significant difference pre- to postbronchodilator (noted only in Absolute $\Delta$ column), or in severe versus moderate subgroups (comparisons of $\%$ pred values and $\Delta \%$ only). ${ }^{\S}: \mathrm{p}=0.08$.

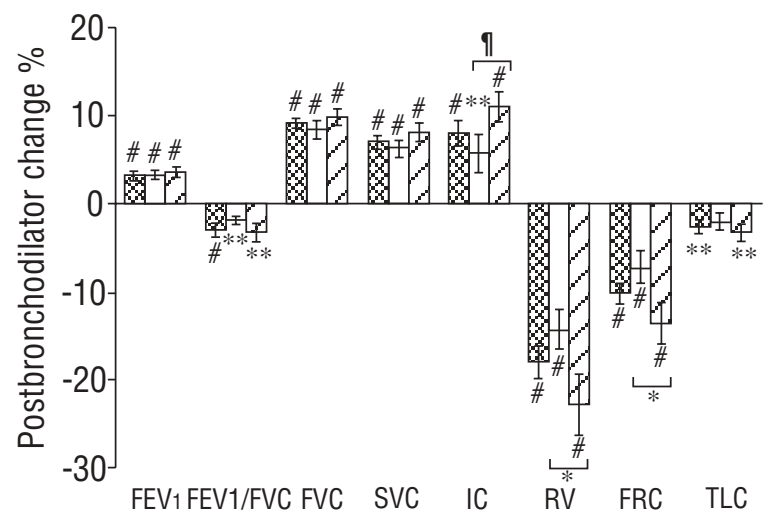

Fig. 1.-Changes in response to salbutamol expressed as percentage of predicted ( $\%$ pred) normal in the chronic obstructive pulmonary disease group as a whole ( $\mathrm{x}=84)$, as well as in its severe $(\mathbb{Z}, n=36)$ and moderate $(\square, n=48)$ subgroups. Values are presented mean $\pm S E M$. $F E V 1$ : forced expiratory volume in one second; FVC: forced vital capacity; SVC: slow vital capacity; IC: inspiratory capacity; RV: residual volume; FRC: functional residual capacity; TLC: total lung capacity. \# : $\mathrm{p}<0.0005$, **: $\mathrm{p}<0.1$, significant changes from pre- to postbronchodilator; *: $\mathrm{p}<0.05$; : $\mathrm{p}=0.08$. 
FEV1/FVC ratio (table 2). All measured lung volumes improved significantly after salbutamol. Postbronchodilator TLC decreased by an average of $0.12 \pm 0.04 \mathrm{~L}$ $(\mathrm{p}<0.01)$, but did not deviate outside the $95 \%$ CI $( \pm 4.5 \%$ pred $)$ of the prebronchodilator measurement in $57 \%$ of patients. The severe group had significantly larger improvements in lung volumes (i.e. decreases in FRC and RV, increases in IC) than the moderate group (table 2, figs. 1 and 2). This greater tendency for patients within the severe subgroup to reduce lung hyperinflation and increase IC was related, in part, to the fact that they were more severely hyperinflated with a significantly reduced baseline IC (table 1): $\triangle$ FRC correlated inversely with prebronchodilator FRC $(\mathrm{r}=-0.37, \mathrm{p}<0.0005)$, $\Delta \mathrm{RV}$ correlated inversely with prebronchodilator $\mathrm{RV}(\mathrm{r}=-0.32, \mathrm{p}<0.01)$, and $\Delta \mathrm{IC}$ correlated inversely with prebronchodilator IC $(\mathrm{r}=-0.24, \mathrm{p}<0.05)$.

$\Delta \mathrm{IC}$ correlated significantly with $\triangle \mathrm{FRC}(\mathrm{r}=-0.60$, $\mathrm{p}<0.0005)$, especially in those patients with no significant change in TLC $(n=48, r=-0.93, p<0.0005)$ (fig. 3$)$. Similarly, $\Delta$ slow vital capacity (SVC) correlated with $\Delta \mathrm{RV}(\mathrm{r}=-0.54, \mathrm{p}<0.0005)$, correlating best in patients with no change in TLC $(\mathrm{n}=48, \mathrm{r}=-0.88, \mathrm{p}<0.0005)$. Compared with $\triangle \mathrm{SVC}, \triangle \mathrm{FVC}$ correlated less strongly with $\Delta \mathrm{RV}(\mathrm{r}=-0.33, \mathrm{p}=0.002)$, even in those patients with no change in TLC $(n=48, r=-0.55, p<0.0005)$. Due to the propensity for a decrease in TLC in the group as a whole, measurements of $\triangle \mathrm{IC}$ and $\triangle \mathrm{SVC}$ underestimated the magnitude of $\triangle F R C$ and $\Delta R V$, respectively. In addition, there were 11 patients without a change in IC but with a reduction in FRC of $\geqslant 10 \%$ pred. In these patients, the reduction in FRC of $0.49 \pm 0.07 \mathrm{~L}$ matched the reduction in TLC of $0.44 \pm 0.06 \mathrm{~L}$.

A significant proportion of subjects improved lung-volume measurements by $\geqslant 10 \%$ pred (fig. 4). Evaluation of plethysmographically-determined $\Delta \mathrm{RV}$ provided the greatest proportion $(61 \%)$ of volume responses $\geqslant 10 \%$ pred, while evaluation of $\Delta \mathrm{IC}$ resulted in the largest proportion (44\%) of changes $\geqslant 10 \%$ pred in spirometric measurements (fig. 2). By combining assessments of all volume changes (FVC, SVC, IC, RV, FRC), $83 \%$ of the group showed an improvement in at least one volume by $\geqslant 10 \%$ pred. Of these 70 patients, 10 improved spirometric volumes alone, 16 improved plethysmographic volumes alone, and the remaining 44 improved spirometric as well as plethysmographic lung volumes. In comparison, the 14 patients without a change of $\geqslant 10 \%$ pred in any measured volume had less baseline hyperinflation ( $\mathrm{FRC}=177 \pm 10$ versus $196 \pm 4 \%$ pred; $\mathrm{p}=0.08$ ), with 10 of these 14 patients belonging to the moderate subgroup. The severe subgroup had a larger proportion of patients with volume changes $\geqslant 10 \%$ pred than the moderate subgroup, especially when examining
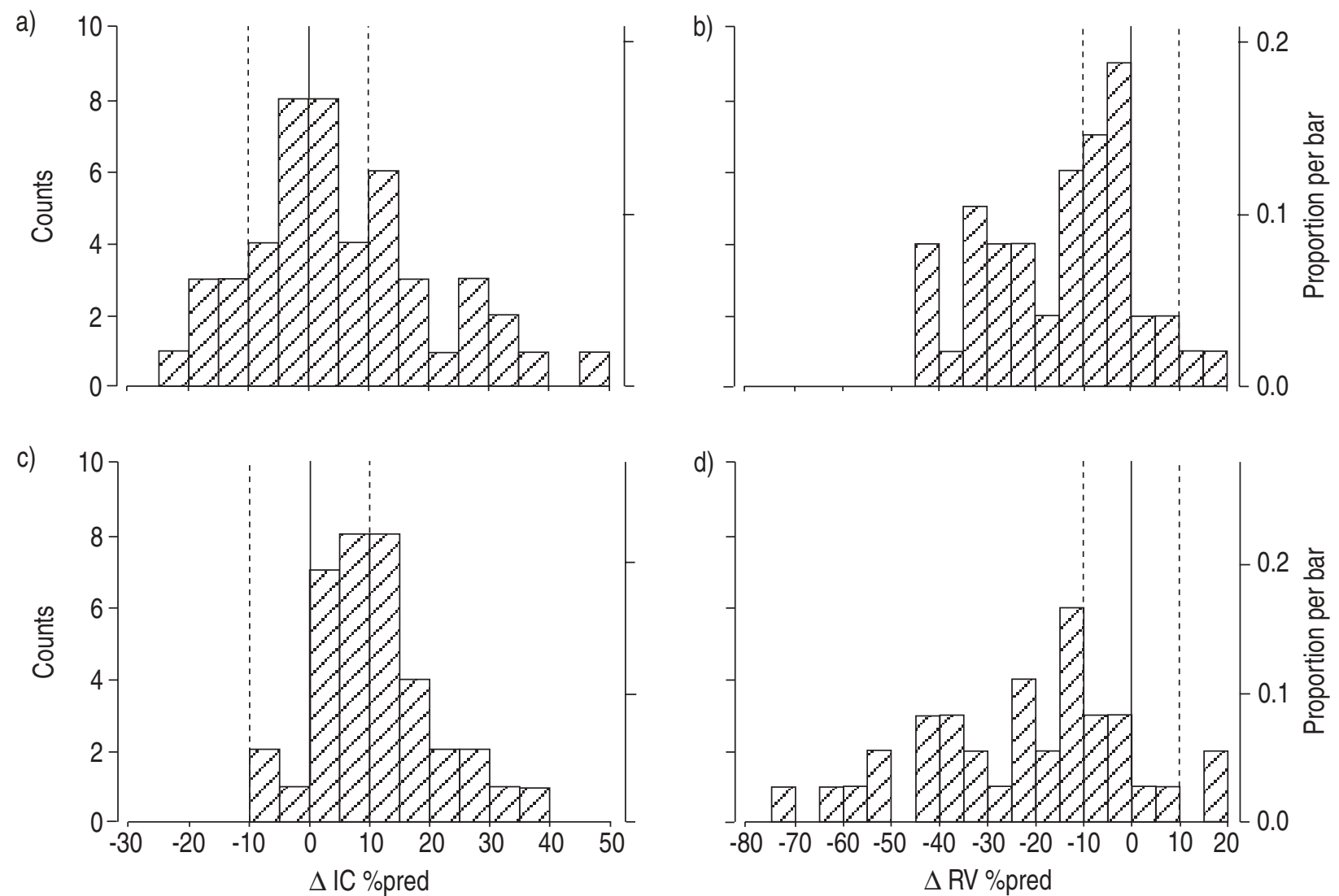

Fig. 2.-Frequency distribution of inspiratory capacity (IC) and residual volume (RV) responses to salbutamol in the severe (c and d; $\mathrm{n}=36)$ and moderate $(\mathrm{a}$ and $\mathrm{b} ; \mathrm{n}=48)$ subgroups with advanced emphysema. Each bar represents a range in change $(\Delta)$ of $5 \%$ pred. "zero" change; -- -: $10 \%$ pred range. 


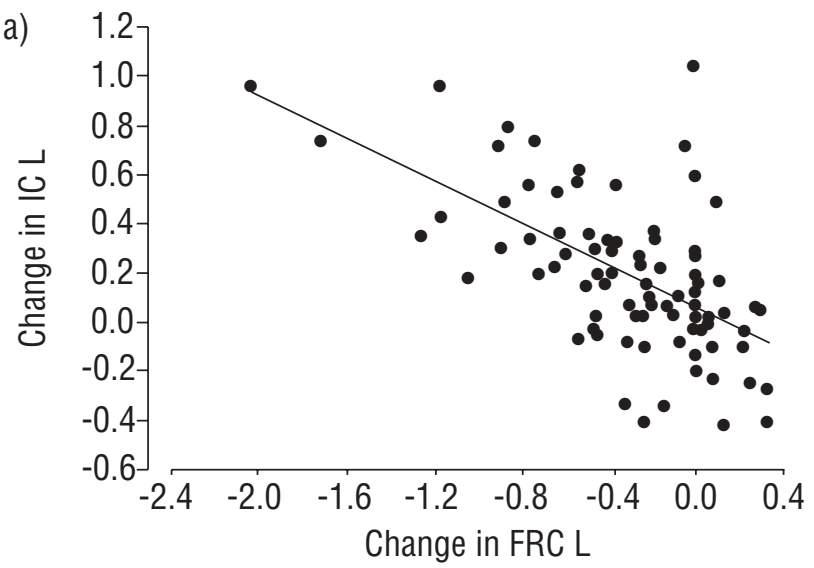

b)

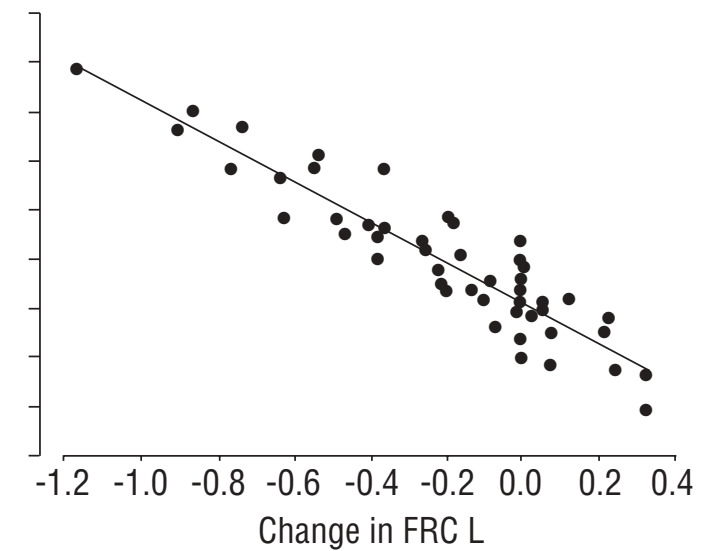

Fig. 3. - Relationship between changes in inspiratory capacity (IC) and functional residual capacity (FRC) in response to salbutamol in a) the chronic obstructive pulmonary disease group as a whole $(\mathrm{n}=84, \mathrm{r}=-0.60, \mathrm{p}<0.0005)$, and within $\mathrm{b})$ the subgroup of patients $(\mathrm{n}=48$, $r=-0.94, p<0.0005)$ with a postbronchodilator total lung capacity that fell within the $95 \%$ confidence interval $( \pm 4.5 \%$ pred $)$ of the prebronchodilator measurement.

changes in operational lung volumes such as IC ( $\mathrm{p}=0.026)$ and FRC ( $\mathrm{p}=0.066)$ (fig. 4).

\section{Discussion}

The novel findings of this study are as follows: 1) a large proportion of patients with advanced emphysema showed significant reductions in lung volumes after salbutamol, despite minimal or no change in the FEV1; 2) RV changes were greater than other volume measurements, and possibly better for the purpose of detecting a significant reduction in air trapping after salbutamol; 3) because of a modest, but consistent reduction in TLC following bronchodilators, changes in IC and VC underestimated changes in FRC and $\mathrm{RV}$, respectively, in a proportion $(43 \%)$ of patients; and 4) the magnitude of volume reduction was significantly larger in patients with more severe emphysema, who had greater baseline lung hyperinflation.

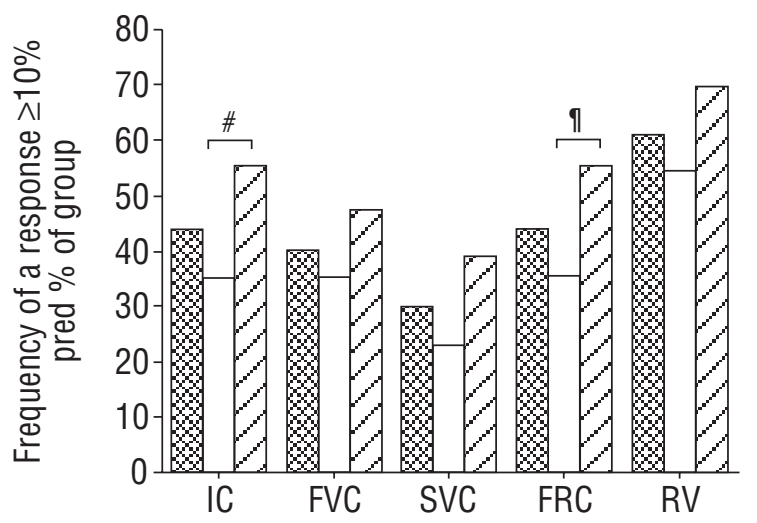

Fig. 4.-Frequency of a bronchodilator response $\geqslant 10 \%$ predicted in various lung volume parameters in the chronic obstructive pulmonary disease group as a whole (网, $n=84$ ), and in its severe $(\mathbb{G}, \mathrm{n}=36)$ and moderate $(\square, \mathrm{n}=48)$ subgroups. IC: inspiratory capacity; FVC: forced vital capacity; SVC: slow vital capacity; FRC: functional residual capacity; RV: residual volume; \# $\mathrm{p}<0.026, ? \mathrm{p}=0.066$.
Current reversibility criteria have been arbitrarily set, such that a postbronchodilator improvement in FEV1 by an amount which exceeds that expected from random variation in the measurement (for a healthy population) represents a significant drug effect [15]. The present study population of patients with advanced, emphysematous COPD showed only trivial changes in FEV1 after salbutamol, suggesting ineffective bronchodilator action. The postsalbutamol FEV1/FVC ratio actually declined significantly, indicating that improved maximal expiratory flow rates reflected increased volume recruitment (i.e. reduced $\mathrm{RV}$ with a resultant increase in VC). The majority of patients showed a significant volume response in the absence of an improvement in FEV1 (fig. 1). In fact, as many as $83 \%$ of the group showed a postsalbutamol change of $\geqslant 10 \%$ pred in at least one of the volume measurements employed.

The addition of body plethysmography identified a further $19 \%$ of patients with a volume response not detected by spirometry alone. The RV response was the most sensitive for detecting a significant reduction in air trapping. In fact, RV decreased by $\geqslant 10 \%$ pred in as many as $61 \%$ of the entire group, and in the majority $(73 \%)$ of those who had an improvement $\geqslant 10 \%$ pred in at least one volume measurement. A reduction in lung volumes following salbutamol must mean enhanced dynamic airway function with more effective lung emptying during tidal and forced expiration, which, in turn, results in reduced gas trapping. Reduced lung hyperinflation implies that patients, although they may remain flow limited, are now able to achieve their required resting alveolar ventilation while breathing at a lower lung volume $[12,23]$.

The ability of a change in IC and VC to reflect changes in FRC and RV, respectively, largely depends on the behaviour of TLC following the use of bronchodilators. Several previous studies have shown that TLC was not changed after the use of a bronchodilator [1, 12, 13, 24, 25]; however, all of these studies had small sample sizes, which raises the possibility of a 
type I error. This is the first study to demonstrate, at least in patients with severe emphysema, that there are small but consistent postbronchodilator reductions in TLC (i.e. by an average of $2.5 \%$ pred or 0.12 $\mathrm{L})$. Thus, changes in IC and VC tended to underestimate concomitant changes in FRC and $\mathrm{RV}$, respectively. Changes in IC accurately reflected a change in FRC in $57 \%$ of the sample (fig. 3). However, significant volume reduction occurred despite an unchanged IC in $13 \%$ of the sample, as a result of a parallel reduction in both TLC and FRC. For practical purposes, an improvement in IC, regardless of the behaviour of TLC, represents a reduction in hyperinflation. Rarely, however, a significant volume reduction can occur in the setting of an unchanged IC following the use of bronchodilators.

Changes in IC were larger and occurred more frequently than changes in VC, suggesting that the former may be a better measure of reduced lung hyperinflation. Changes in SVC correlated better with changes in RV than changes in FVC. In those patients with an unchanged TLC following salbutamol, spirometric FVC may not have accurately reflected the change in RV because of gas and dynamic airway compression effects, which are more pronounced in patients with severe emphysema and hyperinflation. Other discrepancies between spirometric and bodybox measurements may be explained by variation in volume history, expiratory timing during VC comparisons, patient motivation/effort, or testing techniques.

In the group as a whole, the magnitude of lungvolume reduction correlated with the prebronchodilator level of hyperinflation. Compared with the moderate subgroup, reductions in lung hyperinflation were larger (fig. 1) and occurred more frequently (fig. 4) in the severe subgroup with the greatest baseline hyperinflation in this latter group. In this latter group, the average reduction in RV was greater than one-half of a litre. This, in turn, was reflected by larger and more frequent increases in IC in the severe subgroup (figs. 1, 2 and 4).

The relatively diminished volume response to salbutamol in the more moderate COPD subgroup is interesting. TANTUCCI et al. [12], using reduced IC as a marker of hyperinflation, examined bronchodilator responses following higher doses of salbutamol in patients with less severe COPD. They determined that those who did not reduce hyperinflation (i.e. increase IC) after the use of bronchodilators were less likely to have resting EFL, as assessed by the negative expiratory pressure technique; whereas, those with greater resting hyperinflation (i.e. lower IC), and who had a positive-volume response to salbutamol, demonstrated overt EFL at rest. Pellegrino and BRUSASCo [23] arrived at similar conclusions, using partial flow/volume loop analysis to assess expiratory flow limitation. For the patients in the present study who did not demonstrate a volume response to salbutamol, resting FRC was better preserved, possibly indicating less severe EFL at rest, in concurrence with the previously outlined studies. Further studies are required to examine the mechanisms of variability in volume response among patients with emphysema.

The question arises whether volume reductions of this magnitude are likely to translate into clinicallyimportant effects in such patients. Based on current evidence on the effects of $\beta_{2}$-agonists $[1,12,13]$ and anticholinergic agents [2, 3] in advanced COPD, reductions in lung volume, as measured by increases in IC in the order of $10 \%$ pred (i.e. $\sim 0.3-0.4 \mathrm{~L}$ ), translated directly into significant improvements in activity-related dyspnoea and exercise performance. For example, following anticholinergic therapy, the authors have previously shown that a 0.39 -L improvement in resting IC resulted in a $32 \%$ improvement in endurance time using a constant-load cycle exercise protocol [2, 3]. The available evidence, therefore, suggests that a reduction in lung hyperinflation of the magnitude seen in the present study group with advanced emphysema is clinically beneficial. Changes of this magnitude must mean reduced elastic/threshold loading of the inspiratory muscles, reduced constraints on tidal volume expansion during activity, and enhanced neuromechanical coupling of the ventilatory pump [3]. These integrated physiological effects would, if sustained, be expected to improve symptoms, activity levels, and overall health status.

A limitation of this study is that it was not placebo controlled. However, previous studies have shown the absence of any consistent change in lung volumes after placebo [2, 3, 13, 24]. Moreover, the volume changes reported here, particularly in the severe emphysematous group, lay well outside the $95 \%$ CIs of the measurement in the laboratory, and almost certainly reflect a positive effect of the drug. Because the present authors studied a well-characterized group of patients with advanced emphysema, the results may not be generalizable to nonhyperinflated patients with COPD. In fact, those with the least reduction in lung hyperinflation postsalbutamol, had lower levels of lung hyperinflation at baseline.

The authors used low doses of $\beta_{2}$-agonists during a single laboratory reversibility study. The flow and volume responses may well have been amplified by larger dosages of this drug, or by other classes of bronchodilators, alone or in combination. Finally, it is not known whether acute volume reduction after salbutamol in the laboratory can predict a sustained symptomatic benefit in the home for patients taking this drug on a regular basis. Furthermore, placebocontrolled studies with concomitant measurements of exercise endurance and dyspnoea are underway to evaluate the clinical significance of this degree of pharmacological volume reduction.

In summary, traditional criteria for bronchodilator reversibility underestimated a beneficial effect of salbutamol in patients with severe emphysema. Of all the volume measurements, residual volume enabled detection of the greatest proportion of volume responders. Increased inspiratory capacity corresponded directly with reduced operating lung volumes in the majority $(57 \%)$ of patients, while significant volume reduction occurred in the absence of change in inspiratory capacity in only a minority of patients. The magnitude of lung-volume reduction was largest in those with more severe emphysema, and based on available evidence, would be expected to translate into meaningful clinical improvement. The implication of 
these results is that the addition of volume measurements to conventional forced expiratory volume in one second measurements should enhance the assessment of bronchodilator efficacy in advanced hyperinflated chronic obstructive pulmonary disease.

\section{References}

1. Belman MJ, Botnick WC, Shin JW. Inhaled bronchodilators reduce dynamic hyperinflation during exercise in patients with chronic obstructive pulmonary disease. Am J Respir Crit Care Med 1996; 153: 967975.

2. O'Donnell DE, Lam M, Webb KA. Measurement of symptoms, lung hyperinflation and endurance during exercise in chronic obstructive pulmonary disease. $\mathrm{Am}$ J Respir Crit Care Med 1998; 158: 1557-1565.

3. O'Donnell DE, Lam M, Webb KA. Spirometric correlates of improvement in exercise performance after anticholinergic therapy in chronic obstructive pulmonary disease. Am J Respir Crit Care Med 1999; 160: 542-549.

4. O'Donnell DE. Assessment of bronchodilator efficacy in symptomatic COPD: Is spirometry useful? Chest 2000; 117: 42-47.

5. Pride NB, Macklem PT. Lung mechanics in disease. In: AP Fishman, ed. Handbook of Physiology. Section 3, Vol. III, Part 2: The Respiratory System. Bethesda, MD, American Physiological Society, 1986; pp. 659-692.

6. Hyatt RE. Expiratory flow limitation. J Appl Physiol 1983; 55: 1-8.

7. Carlson DJ, Ries AL, Kaplan RM. Predictors of maximum exercise tolerance in patients with COPD. Chest 1991; 100: 307-311.

8. Bauerle O, Chrusch CA, Younes M. Mechanisms by which COPD affects exercise tolerance. Am J Respir Crit Care Med 1998; 157: 57-68.

9. O'Donnell DE, Webb KA. Exertional breathlessness in patients with chronic airflow limitation: the role of lung hyperinflation. Am Rev Respir Dis 1993; 148: 1351-1357.

10. Diaz $\mathrm{O}$, Villafranco $\mathrm{C}$, Ghezzo $\mathrm{H}$, et al. Exercise tolerance in COPD patients with and without tidal expiratory flow limitation at rest. Eur Respir J 2000; 16: $269-275$.

11. O'Donnell DE, Revill SM, Webb KA. Dynamic hyperinflation and exercise intolerance in COPD. $\mathrm{Am}$ J Respir Crit Care Med 2001; 164: 770-777.

12. Tantucci C, Duguet A, Similowski T, Zelter M, Derenne J-P, Milic-Emili J. Effect of salbutamol on dynamic hyperinflation in chronic obstructive pulmonary disease patients. Eur Respir J 1998; 12: 799-804.

13. Ramirez-Venegas A, Ward J, Lentine T, Mahler DA. Salmeterol reduces dyspnea and improves lung function in patients with COPD. Chest 1997; 112: 336-340.

14. Pauwels RA, Buist AS, Calverley PMA, Jenkins CR, Hurd SS, on behalf of the GOLD Scientific Committee. Global strategy for the diagnosis, management and prevention of chronic obstructive pulmonary disease: NHLBI/WHO Global initiative for chronic obstructive lung disease (GOLD) workshop summary. Am J Respir Crit Care Med 2001; 163: 1256-1276.

15. Siafakas NM, Vermeire P, Pride NB, et al. ERS consensus statement: optimal assessment and management of chronic obstructive pulmonary disease (COPD). Eur Respir J 1995; 8: 1398-1420.

16. American Thoracic Society. Standards for the diagnosis and care of patients with chronic obstructive pulmonaray disease (COPD) and asthma. Am Rev Respir Dis 1987; 136: 225-244.

17. American Thoracic Society. Standardization of spirometry - 1994 update. Am J Respir Crit Care Med 1995; 152: $1107-1136$.

18. Shore SA, Huk O, Mannix S, Martin JG. Effect of panting frequency on the plethysmographic determination of thoracic gas volume in chronic obstructive pulmonary disease. Am Rev Respir Dis 1983; 128: 54-59.

19. Morris JF, Koski A, Temple WP, Claremont A, Thomas DR. Fifteen year interval spirometric evaluation of the Oregon predictive equations. Chest 1988; 93: $123-127$.

20. Crapo RA, Morris AH, Clayton PD, Nixon CR. Lung volumes in healthy nonsmoking adults. Bull Europ Physiopath Respir 1982; 18: 419-425.

21. Burrows B, Kasik JE, Niden AH, Barclay WR. Clinical usefulness of the single-breath pulmonary diffusing capacity test. Am Rev Respir Dis 1961; 84: 789-806.

22. Newton M, O'Donnell DE, Forkert L. Response of lung volumes to inhaled albuterol in a large population of patients with severe hyperinflation. Am J Respir Crit Care Med 2000; 161: A209.

23. Pellegrino R, Brusasco V. Lung hyperinflation and flow limitation in chronic airway obstruction. Eur Respir J 1997; 10: 543-549.

24. Hughes JA, Tobin MJ, Bellamy D, Hutchison DCS. Effects of ipratropium bromide and fenoterol aerosols in pulmonary emphysema. Thorax 1982; 37: 667-670.

25. Lavietes $\mathrm{MH}$, Taylor DW. Determination of static pulmonary volumes after bronchodilator therapy. Chest 1979; 76: 425-428. 